$\begin{array}{llllllllllllll}\text { A C T A } & \text { C H E M I C A } & \text { S C A N D I N A V I C A } & 23 & \text { (1969) } & 846-858\end{array}$

\title{
Separation of Porphyrins by Multiple Liquid-Liquid Partition
}

\author{
NILS ELLFOLK, PAAVO HYNNINEN and GUNNEL SIEVERS
}

Department of Biochemistry, University of Helsinki, Helsinki 17, Finland

\begin{abstract}
Crude preparations of proto-, meso-, deutero-, and hematoporphyrins have been purified by liquid-liquid partition in a formic acid-chloroform solvent system. Stepwise $\mathrm{pH}$ gradient elution was used to separate a mixture of proto-, meso-, deutero-, 2(4)-vinyl$4(2)$-hydroxyethyldeutero-, and hematoporphyrins in 50 tubes. The advantages of this method are discussed. The properties of the aqueous formic acid-chloroform solvent system have been compared in detail with those of the conventional aqueous hydrochloric acid-diethyl ether solvent system. The most serious limitation of both solvent systems from the preparative point of view seems to be the association of porphyrin molecules in the organic phase. This phenomenon is probably the main reason for excessive broadening of the concentration zones which leads to impaired resolution of the components. The broadening can be diminished by lowering the porphyrin concentration in the system.
\end{abstract}

$\mathrm{T}_{\mathrm{d}}$ he multiple liquid-liquid partition technique known as "countercurrent distribution" (CCD) has been used in a number of investigations ${ }^{1-6}$ to separate free porphyrins. The diethyl ether-aqueous hydrochloric acid solvent system introduced by Willstätter and Mieg ${ }^{7}$ and later studied in detail by Zeile and Rau ${ }^{8}$ (see also Herbert ${ }^{9}$ ) has been used almost exclusively. However, this solvent system has several drawbacks which make it a less suitable solvent system for quantitative liquid-liquid partition investigations.

In this communication the use of the aqueous formic acid-chloroform (FC) solvent system for separating free dicarboxylic porphyrins is described and the results are compared with those obtained with the ether-aqueous hydrochloric acid solvent system. Instead of the conventional Craig machine, the apparatus developed by Hietala ${ }^{10}$ was used.

\section{THEORETICAL}

Partition behaviour of porphyrins in an organic solvent-aqueous acid system. In the ether-aqueous hydrochloric acid solvent system at equilibrium dicarboxylic porphyrins are mainly present as dications in the aqueous phase and 
as neutral compounds in the organic phase.$^{8,9}$ An identical separation of neutral and cationic porphyrins was found to occur also in the FC system. By taking into account the two protolytic equilibria

$$
\mathrm{PH}_{2}+\mathrm{H}^{+} \stackrel{K_{3}}{\rightleftharpoons} \mathrm{PH}_{3}{ }^{+}, \quad \mathrm{PH}_{3}{ }^{+}+\mathrm{H}^{+} \stackrel{K_{4}}{\rightleftharpoons} \mathrm{PH}_{4}^{2+}
$$

where $\mathrm{PH}_{2}$ denotes the neutral form of porphyrin, $\mathrm{PH}_{3}{ }^{+}$and $\mathrm{PH}_{4}^{2+}$ denote the mono- and dications, and $K_{3}$ and $K_{4}$ their acid dissociation constants, ${ }^{11}$ the following relationship is obtained:

$$
\log \frac{\left(\mathrm{PH}_{2}\right)}{\left(\mathrm{PH}_{4}^{2+}\right)}=2 \mathrm{pH}-\left(\mathrm{p} K_{3}+\mathrm{p} K_{4}\right)
$$

By taking $\left(\mathrm{PH}_{2}\right) /\left(\mathrm{PH}_{4}^{2+}\right)$ approximately equal to the experimental partition coefficient, $K=c_{\text {org }} / c_{\text {aq }}$, where $c_{\text {org }}$ and $c_{\mathrm{aq}}$ are the total porphyrin concentrations in the organic and aqueous acid phases, respectively, the following equation is obtained:

$$
\log K=2 \mathrm{pH}-\text { constant. }
$$

Thus, $\log K$ should be a linear function of $\mathrm{pH}$, the slope of the line should be 2 , and the intercept of the line should be essentially equal to $\mathrm{p} K_{3}+\mathrm{p} K_{4}$.

However, this relationship may not be strictly valid experimentally for the following reasons. Substitution of $K$ for $\left(\mathrm{PH}_{2}\right) /\left(\mathrm{PH}_{4}{ }^{2+}\right)$ may be a crude approximation, because $\mathrm{PH}_{2}$ may dissolve to an appreciable extent in the aqueous acid phase or $\mathrm{PH}_{4}{ }^{2+}$ in the organic phase depending on the mutual solubility of the phases. Other reactions such as the association of neutral porphyrin molecules in the organic phase may also take place.

Calculation of theoretical distribution curves. The concentration profiles in the apparatus of Hietala are generally in accord with the theory of Martin and Synge. ${ }^{12}$ When the compounds are still in the train, the theoretical distribution curves may be calculated according to the Poisson distribution ${ }^{10}$ if the number of tubes through which the concentration maximum has been eluted $\left(r_{\max }\right)$ is not very large. In other cases the assumption of a normal distribution is convenient (as a rule the Poisson distribution can be replaced by the normal distribution if $r_{\max } \geq 30$ ).

In the approximation the abscissa of the standard normal curve, i.e., the parameter $t$, is given by the equation:

$$
t=\frac{r-r_{\max }}{\sqrt{r_{\max }}}
$$

where $r$ is the tube number of the unknown concentration and $r_{\max }$ the tube number of the maximum concentration $(r$ is numbered, $r=0,1,2, \ldots, u$, beginning from the loading end of the tube row). The corresponding ordinate of the standard normal curve, $f(t)$, is obtained from a statistical table. The unknown absorbance, $A$, may then be calculated directly from:

$$
A=A_{\max } \cdot f(t) / 0.399
$$

Acta Chem. Scand. 23 (1969) No. 3 
In the case of the elution analysis, the parameter $t$ is given by the equation:

$$
t=\frac{\left(V-V_{\max }\right) \sqrt{u}}{V_{\max }}
$$

where $V$ is the elution volume of the unknown concentration, $V_{\max }$ the elution volume of the maximum concentration, and $u$ the number of tubes through which the maximum concentration has been eluted.

Calculation of the partition coefficient. Reliable values for the partition coefficient can be obtained from the multiple partition data as follows. The operational partition coefficient, $k$, defined as $c_{\mathrm{m}} / c_{\mathrm{s}}$, where $c_{\mathrm{m}}$ and $c_{\mathrm{s}}$ are the porphyrin concentrations in the mobile and the stationary phases, respectively, in a partition unit, is given by the equation:

$$
k=\frac{v_{\mathrm{s}}}{\left(V_{\mathrm{m}} / r_{\max }\right)-v_{\mathrm{m}}}=\frac{v_{\mathrm{s}}}{\left(V_{\max } / u\right)-v_{\mathrm{m}}}
$$

where $v_{\mathrm{m}}$ and $v_{\mathrm{s}}$ are the volumes of the mobile and the stationary phases, respectively, in the partition unit, and $V_{\mathrm{m}}$ is the volume of mobile phase needed to transfer the maximum concentration to the tube $r=r_{\max }$. The actual partition coefficient, $K$, which is defined as $c_{\mathrm{org}} / c_{\mathrm{aq}}$, is equal to $k$ when the lower (chloroform) phase is mobile, and equal to $1 / k$ when the upper (aqueous acid) phase is mobile.

\section{EXPERIMENTAL}

\section{Materials}

Crystalline protohemin $I X$ was a commercial preparation from Sigma Chemical Co.

Protoporphyrin $I X$ was prepared from the protohemin by the method of Morell and Stewart. ${ }^{3}$

Mesoporphyrin IX was prepared according to Muir and Neuberger ${ }^{14}$ by catalytic reduction of protoporphyrin using a Pd catalyst of the polyvinyl type described by Rampino and Nord. ${ }^{15}$

Deuteroporphyrin IX was prepared by heating protohemin with resorcinol at 170 $180^{\circ} \mathrm{C}$ for 20 min..$^{16}$ Iron was removed by treating the crude reaction mixture directly with iron powder, acetic acid and $\mathrm{HCl} \cdot{ }^{17}$

Hematoporphyrin IX was a commercial preparation of hematoporphyrin dihydrochloride (Koch-Light \& Co.). The spectrum of the preparation gave no indication of 2(4)-vinyl-4(2)-hydroxyethyldeuteroporphyrin.

2(4)-Vinyl-4(2)-hydroxyethyldeuteroporphyrin $I X$ was obtained from the crude deuteroporphyrin preparation by a simple purification procedure described below.

The solvents used were analytical grade reagents. Chloroform was washed three times with distilled water immediately before use.

\section{E q u i p m e n t}

For multiple liquid-liquid partition, the apparatus developed by Hietala ${ }^{10}$ was used. This apparatus was manufactured by Karppinen Oy (Sahaajank. 7, Helsinki, Finland), and contained 200 glass units (unit volume $13.5 \mathrm{ml}$ ). Teflon tubing was used to connect the tubes to each other in series and to convey liquids to and from the glass tube train. A micropump giving a flow rate of $1-2 \mathrm{ml} / \mathrm{min}$ and a commercial fraction collecter (LKB) were used as ancillary equipment. 
A Beckman Recording Spectrophotometer Model DK-1 was used to measure the porphyrin spectrograms and a Beckman DU Spectrophotometer to measure single absorbances.

A Radiometer PHM 4c pH meter was used to measure hydrogen ion concentrations at $20^{\circ} \mathrm{C}$. The $\mathrm{pH}$ meter was calibrated with a phthalate buffer.

\section{Selection of the solvent system}

In order to find a solvent system suitable for the fractionation of dicarboxylic porphyrins, preliminary experiments were carried out in a separatory funnel. Formic acid was selected instead of hydrochloric acid, because the former is a considerably weaker acid than the latter. As nonpolar solvent, petroleum ether, benzene, and toluene were tested, but these were poor solvents. Especially in the case of petroleum ether, porphyrin had a strong tendency to accumulate at the interface of the solvent system and on the wall of funnels even when the concentration of formic acid in the aqueous phase was relatively high. However, chloroform in combination with aqueous formic acid was found to have a high capacity to dissolve porphyrins.

\section{Operation of the distribution train}

Determination of the phase ratio. In order to obtain accurate values for the partition coefficient (eqn. (6)), the phase ratio, $\alpha=v_{\mathrm{m}} / v_{\mathrm{s}}$, must be accurately measured. For this purpose all the tubes to be used in the separation process were first filled with the stationary phase only and the volume of solvent needed was measured. The tubes were then turned to a position determined by the desired phase ratio. Keeping the tubes in this position, the mobile phase was pumped in until the stationary phase no longer emerged from the train. The stationary phase was collected and measured. From these data $v_{\mathrm{m}}, v_{\mathrm{s}}$, and the exact phase ratio could be calculated.

Selection of operation conditions. Because the separation is a maximum in the MartinSynge distribution when $v_{\mathrm{m}} / v_{\mathrm{s}}=0$, the lowest possible phase ratio must be selected in order to use the distribution train effectively for separation. When the aqueous acid phase was mobile, the lower limit of the phase ratio was found to be about 0.18 , and when the chloroform phase was mobile, it was about 0.35 . At lower values than these, the phase ratio did not remain constant. A shaking frequency of 29 cycles per minute, an amplitude of $\pm 45^{\circ}$, and a flow rate of $1-2 \mathrm{ml} / \mathrm{min}$ were used in these studies. These values were selected as tentative operating conditions for the fractionation experiments.

Fractionation. At the beginning of a fractionation, the porphyrin mixture was dissolved in sufficiently large volumes of chloroform and aqueous formic acid phases in a separatory funnel, but keeping the phase ratio about the same as was to be used in the distribution train. The first tubes of the train were emptied of solvents, and the porphyrin mixture was loaded into these tubes. Generally the first $1-7$ tubes were needed depending on the amount and nature of the porphyrin mixture. After this the located tubes were stoppered, and the fractionation was started.

Two different methods were used for developing and analyzing the concentration profiles. In the first method, the process was stopped when the compounds were still in the train, aliquots of the upper or the lower phases were taken from the tubes and their absorbances measured at the Soret band wavelength. This method corresponds to the "fundamental distribution" process of the CCD. In the second method, the compounds were eluted from the train, the effluent was collected in fractions in an automatic fraction collector, and the fractions were analyzed spectrophotometrically. This method corresponds to the "elution analysis" of chromatography or the "single withdrawal" of the CCD. The second method was found to be more convenient and was used in the later fractionation experiments.

Acta Chem. Scand. 23 (1969) No. 3 


\section{RESULTS}

Purification of crude porphyrin preparations. The porphyrin preparations were first purified separately in tentative fractionation experiments described in the following. The same concentration of formic acid (4.34 M, pH 1.22) in the upper phase was used in these experiments. The results of the purification of a crude mesoporphyrin preparation is presented in Fig. 1. Two components could easily be completely separated from each other by means of only fifty tubes. The more rapidly moving component (1) had a partition coefficient $K_{(1)}=1.65$. This is of the same order as that expected for mesoporphyrin IX (according to partition studies carried out with mesoporphyrin IX in the diethyl ether-hydrochloric acid systems, $K$ is about 4 when $[\mathrm{HCl}]=0.06$ $\mathrm{N}^{9}$ ). The component (2) remained at the loading end of the appratus. This porphyrin was practically insoluble in the aqueous acid ( $\mathrm{pH} 1.22)$, but dis-

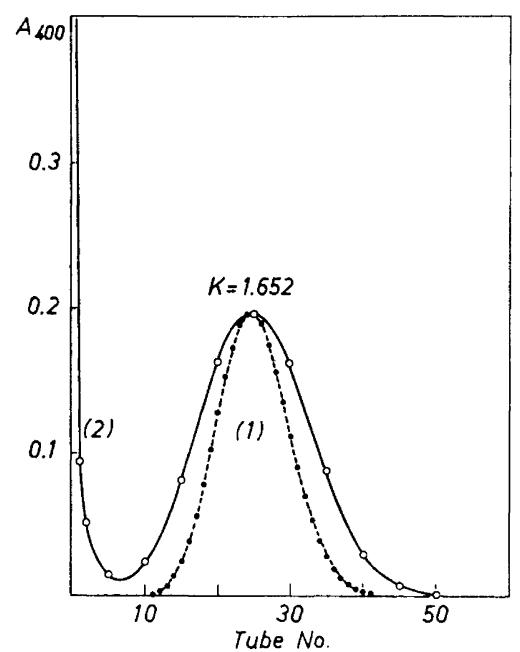

Fig. 1. Purification of the mesoporphyrin IX preparation. Solvent system: $4.34 \mathrm{M}$ $\mathrm{HCOOH}\left(\mathrm{pH} \mathrm{1.22)}-\mathrm{CHCl}_{3} .0 .27 \mathrm{mg}\right.$ of the crude mesoporphyrin preparation was dissolved in $23 \mathrm{ml}$ of $\mathrm{CHCl}_{3}$ and $4.0 \mathrm{ml}$ of aqueous $\mathrm{HCOOH}$, and the mixture was loaded into the two first tubes of the distribution apparatus. The number of tubes used in the process was $50 . v_{\mathrm{m}}=2.0 \mathrm{ml}$ (aqueous $\mathrm{HCOOH}), v_{\mathrm{S}}=11.5 \mathrm{ml}\left(\mathrm{CHCl}_{3}\right)$, $V_{\mathrm{m}}=525 \mathrm{ml}$, flow rate $=2 \mathrm{ml} / \mathrm{min}$. (O) experimental curve, obtained by measuring $A_{400}$ of the lower phases. (O), theoretical curve, calculated according to the properties of the Poisson distribution. (1) Mesoporphyrin IX, (2) an unknown porphyrin.

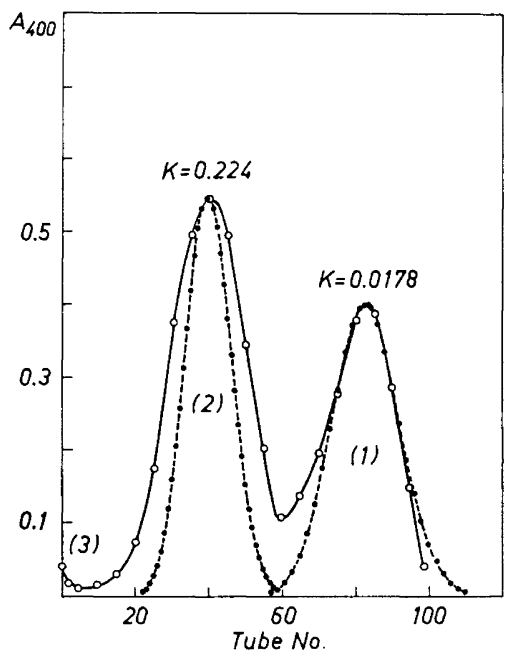

Fig. 2. Purification of the deuteroporphyrin IX preparation. Solvent system: $4.34 \mathrm{M}$ $\mathrm{HCOOH}(\mathrm{pH} 1.22)-\mathrm{CHCl}_{3} \cdot 2.34 \mathrm{mg}$ of the crude deuteroporphyrin preparation was dissolved and loaded into the distribution apparatus as in Fig. 1. Number of tubes used in the process was 100. $v_{\mathrm{m}}=2.0 \mathrm{ml}$ (aqueous $\mathrm{HCOOH}), v_{\mathrm{s}}=11.5 \mathrm{ml}\left(\mathrm{CHCl}_{3}\right)$, $V_{\mathrm{m}}=183 \mathrm{ml}$, flow rate $=2 \mathrm{ml} / \mathrm{min}$. (O), experimental curve, obtained by taking aliquots $(0.2 \mathrm{ml})$ of the upper phases, diluting these to $3.0 \mathrm{ml}$ with the aqueous $\mathrm{HCOOH}$, and measuring $A_{400}$. (O), theoretical curve, calculated according to the properties of the Poisson distribution. (1) 2(4) - Vinyl - 4(2) - hydroxyethyldeuteropor. phyrin IX, (2) deuteroporphyrin IX, (3) an unknown porphyrin.

Acta Chem. Scand. 23 (1969) No. 3 
solved well in chloroform. By taking into account the absorbances of the lower phases of the two first tubes, $A=1.25(r=0)$ and $A=0.094(r=1)$, it can be estimated that $K_{(2)} \gg 45.5$. In spite of their greatly different partition properties, the two components resembled each other in their absorption spectra. Component (1) had absorption maxima at 398, 498, 532, 566, 594, and $620 \mathrm{~nm}$ and component (2) at 400,500,533, 566, and $620 \mathrm{~nm}$ in chloroform. The porphyrin preparation contained $78 \%$ of component (1) (mesoporphyrin IX) and $22 \%$ of component (2), an unknown porphyrin.

Fig. 2 shows the result of the purification of the crude deuteroporphyrin preparation. In this case three components could be separated from each other by means of one hundred tubes. The most rapidly moving component (1) had a partition coefficient $K_{(1)}$ equal to 0.0178 and was thus only slightly soluble in the chloroform phase. The absorption maxima of this porphyrin were at $402,501,536,569,603$, and $624 \mathrm{~nm}$ in chloroform. The additional absorption band at $603 \mathrm{~nm}$ was unusually strong. Component (1) was identified as 2(4)-vinyl-4(2)-hydroxyethyldeuteroporphyrin IX on the basis of its partition behaviour and spectroscopic properties. Component (2) had a parti-

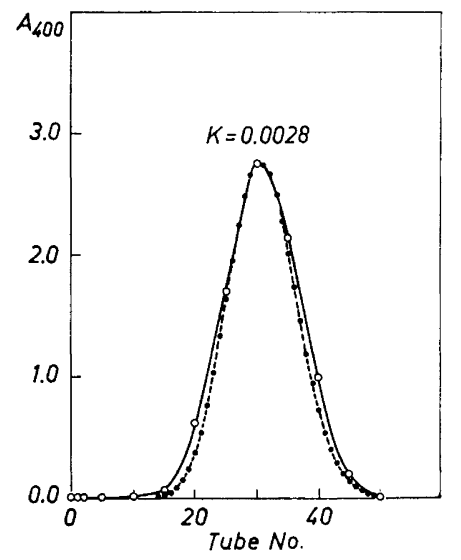

Fig. 3. Purification of the hematoporphyrin IX preparation. Solvent system: $4.34 \mathrm{M}$ $\mathrm{HCOOH}(\mathrm{pH} \mathrm{1.22})-\mathrm{CHCl}_{3} .5 .20 \mathrm{mg}$ of the hematoporphyrin was dissolved and loaded into the distribution apparatus as in Fig. 1. Number of tubes used in the process was $50 . v_{\mathrm{m}}=2.0 \mathrm{ml}$ (aqueous $\mathrm{HCOOH}$ ), $v_{\mathrm{s}}=11.5$ $\mathrm{ml}\left(\mathrm{CHCl}_{3}\right), \quad V_{\mathrm{m}}=63 \mathrm{ml}$, flow rate $=1$ $\mathrm{ml} / \mathrm{min}$. (O), experimental curve, obtained by taking aliquots $(0.1 \mathrm{ml})$ of the upper phases, diluting these to $3.0 \mathrm{ml}$ with the aqueous $\mathrm{HCOOH}$, and measuring $A_{400}$. (O), theoretical curve, calculated according to the properties of the Poisson distribution.

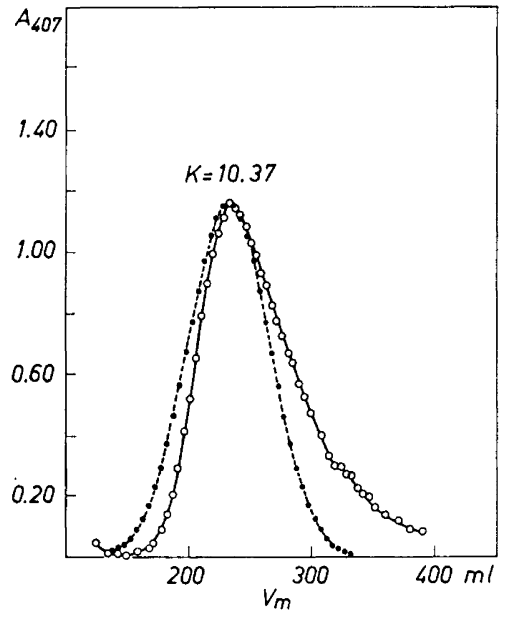

Fig. 4. Distribution of protoporphyrin IX. Solvent system: 4.0 M HCOOH (pH 1.25) $-\mathrm{CHCl}_{3} .0 .40 \mathrm{mg}$ of protoporphyrin preparation was dissolved in $26 \mathrm{ml}$ of $\mathrm{CHCl}_{3}$ and $68 \mathrm{ml}$ of aqueous $\mathrm{HCOOH}$, and the mixture was loaded into the first seven tubes of the distribution apparatus. Number of tubes used in the process was 50 . $v_{\mathrm{m}}=3.8 \mathrm{ml}\left(\mathrm{CHCl}_{3}\right), v_{\mathrm{s}}=\mathbf{9 . 7} \mathrm{ml}$ (aqueous $\mathrm{HCOOH}$ ), flow rate $=2 \mathrm{ml} / \mathrm{min}$. (O), experimental curve, obtained by measuring $A_{407}$ of the effluent fractions. (0), theoretical curve, calculated by means of eqns.
(4) and

(5) $\begin{gathered}\left(V_{\max }=232\right. \\ \left.A_{\max }=1.16\right)\end{gathered}$

Acta Chem. Scand. 23 (1969) No. 3 
tion coefficient $K_{(2)}=0.224$ and absorption maxima at $399,497,530,566$, 593 , and $620 \mathrm{~nm}$ in chloroform. From these properties component (2) was identified as deuteroporphyrin IX. The third component (3), which remained at the loading end of the apparatus, resembled closely component (2) in the mesoporphyrin preparation. It had a partition coefficient $K_{(3)} \gg 15.7$ and absorption maxima at 400, 500, 533, 568-570, and $621 \mathrm{~nm}$ in chloroform. The deuteroporphyrin preparation contained $29 \%$ of component (1) (2(4)vinyl-4(2)-hydroxyethyldeuteroporphyrin IX), $51 \%$ of component (2) (deuteroporphyrin IX) and $20 \%$ of component (3) (an unknown porphyrin).

Fig. 3 shows the results of the purification of the hematoporphyrin preparation. The single compound had a partition coefficient $K=0.0028$ and absorption maxima at 402,502,535, 570,597, and $623 \mathrm{~nm}$ in chloroform. Although the deviation from theory is negligible in this case, the low value of $K$ makes it a less reliable evidence of purity.

The purification of the protoporphyrin preparation was studied in the same solvent system as was used in the previous experiments. The chloroform phase was selected as the mobile solvent. Fig. 4 presents an elution analysis when only 50 tubes were used. The experimental curve deviates considerably

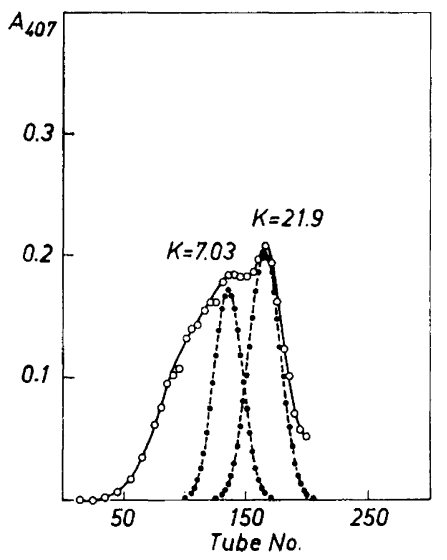

Fig. 5. Distribution of protoporphyrin IX. Solvent system: 4.34 M HCOOH (pH 1.22) $-\mathrm{CHCl}_{3}$. $0.40 \mathrm{mg}$ of protoporphyrin was dissolved and loaded into the distribution apparatus as described in the text to Fig. 4. Number of tubes used in the process was 200. $v_{\mathrm{m}}=3.8 \mathrm{ml}\left(\mathrm{CHCl}_{3}\right), v_{\mathrm{s}}=9.7 \mathrm{ml}$ (aqueous $\mathrm{HCOOH}$ ), $V_{\mathrm{m}} \simeq 700 \mathrm{ml}$, flow rate $=2 \mathrm{ml} / \mathrm{min}$. (O), experimental curve, obtained by measuring $A_{407}$ of the lower phases. (O), theoretical curve, calculated by means of eqns. (3) and (4). (1) and (2) are different forms of protoporphyrin IX.

from the theoretical one. Fig. 5 shows the situation when all the 200 tubes of the apparatus were used. The concentration profile clearly reveals the presence of two (or more) components in the zone. However, their resolution is only partial, and in addition large deviations from theory are observed. The differences between the absorption spectra of the lower phases of the front and the back ends of the zone were negligible. Both spectra were closely similar to that of protoporphyrin (absorption maxima at 408, 508, 543, 577, 606 , and $632 \mathrm{~nm}$ in chloroform). On continuing the process using the single withdrawal procedure, the resolution of the components was not improved. On the contrary, it was somewhat impaired, for the broadening (tailing) of the zone increased. It seems that the porphyrin existed in the lower phase in two different forms which were in equilibrium with each other. The more 
rapidly moving component (1) is probably the associated form (dimer or polymer), and component (2) the unassociated form (monomer) of protoporphyrin.

The effect of increased formic acid concentration on the distribution of protoand mesoporphyrins. The foregoing experiments showed that association of porphyrin molecules may occur in the lower phase. This phenomenon seems to be the main reason for the observed excess broadening of the concentration zones. However, it was thought possible to avoid this by using a higher concentration of formic acid in the solvent system. In order to elucidate this question, some additional distribution studies were carried out with protoand mesoporphyrins.

By increasing the concentration of formic acid $(7.32 \mathrm{M}, \mathrm{pH} 0.835)$ in the upper phase, a decrease in the deviation from theory was observed in the distribution of protoporphyrin (Fig. 6). A slight inhomogeneity was observed in the front of the principal concentration zone.

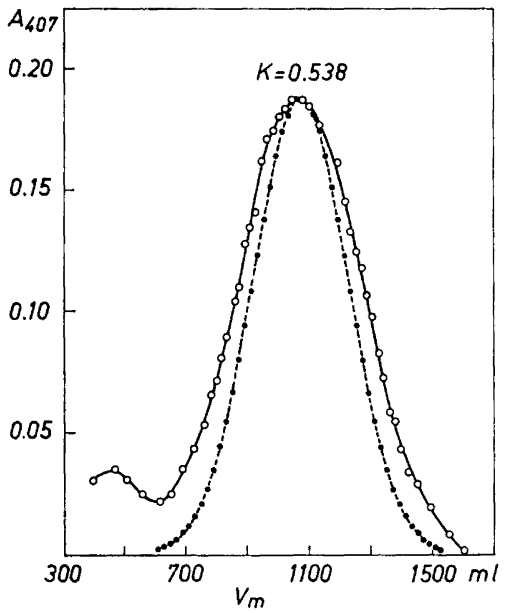

Fig. 6. Distribution of protoporphyrin IX. Solvent system: 7.32 M HCOOH (pH 0.835) $-\mathrm{CHCl}_{3}$. The experimental conditions were the same as in Fig. 4. (O), experimental curve, obtained by measuring $A_{107}$ of the effluent fractions. (O), theoretical curve, calculated by means of eqns. (4) and (5) $\left(V_{\max }=1070 \mathrm{ml}, u=49, A_{\max }=0.1875\right)$.

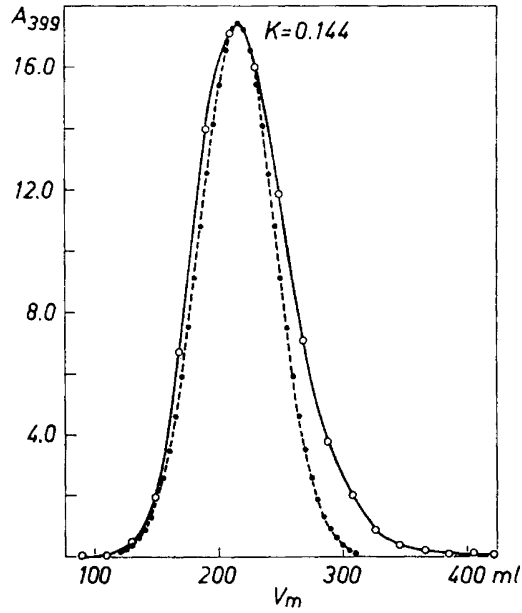

Fig. \%. Distribution of mesoporphyrin IX. Solvent system: 7.32 M HCOOH (pH 0.835) $-\mathrm{CHCl}_{3}$. $2.10 \mathrm{mg}$ of purified mesoporphyrin was dissolved in $75 \mathrm{ml}$ of $\mathrm{CHCl}_{3}$ and $19 \mathrm{ml}$ of aqueous $\mathrm{HCOOH}$ and the mixture was loaded into the first seven tubes of the distribution apparatus. Number of tubes used in the process was $50 . v_{\mathrm{m}}=2.8 \mathrm{ml}$ (aqueous $\mathrm{HCOOH}), v_{\mathrm{s}}=10.7 \mathrm{ml}\left(\mathrm{CHCl}_{3}\right)$, flow rate $=2 \mathrm{ml} / \mathrm{min}$. (O), experimental curve obtained by measuring $A_{399}$ of the effluent fractions. (O), theoretical curve calculated by means of eqns. (4) and (5)

$\left(V_{\max }=215 \mathrm{ml}, u=49, A_{\max }=17.4\right)$. 
Mesoporphyrin was also studied at the same concentration of formic acid in the lower phase (7.32 M, pH 0.835). The upper phase was selected as the mobile solvent. The results of the experiment are shown in Fig. 7. Excessive broadening of the concentration zone is observed in this case, too.

Simultaneous separation of several porphyrins. On the basis of the foregoing experiments it was evident that the simultaneous separation of several dicarboxylic porphyrins including protoporphyrin would hardly be possible at a constant formic acid concentration in the upper phase. In order to avoid excessive broadening of the concentration zone in the case of protoporphyrin, the $\mathrm{pH}$ of the upper phase should be below 1.0. However, at this $\mathrm{pH}$ value, hemato-, 2(4)-vinyl-4(2)-hydroxyethyldeutero-, and deuteroporphyrins remain practically completely in the upper phase, and very little partition would occur, which means that these porphyrins could not be separated from each other. In order to make possible the simultaneous separation of several porphyrins, stepwise $\mathrm{pH}$ gradient elution was adopted.

The simultaneous separation of five dicarboxylic porphyrins using stepwise $\mathrm{pH}$ gradient elution was studied (Fig. 8). The upper phase was chosen as the

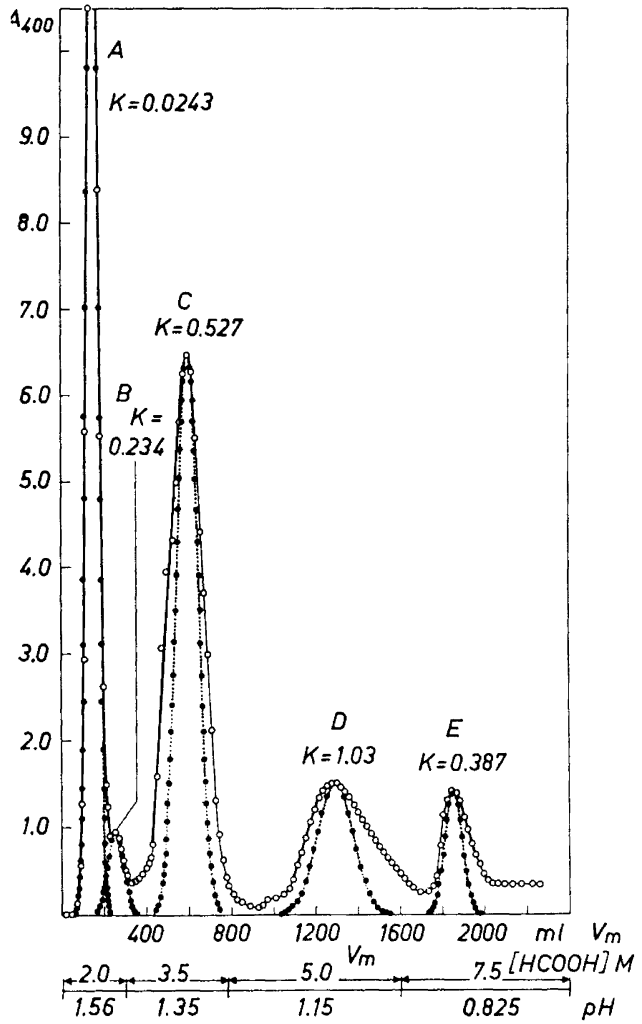

Fig. 8. Simultaneous separation of several porphyrins. Solvent system: aqueous $\mathrm{HCOOH}$ (molarity and $\mathrm{pH}$ varied) $-\mathrm{CHCl}_{3} .2 .30 \mathrm{mg}$ of hemato-, $0.20 \mathrm{mg}$ of 2(4)-vinyl-4(2)-hydroxyethyldeutero-, 1.20 $\mathrm{mg}$ of deutero-, $0.83 \mathrm{mg}$ of meso-, and 0.80 $\mathrm{mg}$ of protoporphyrin were dissolved in 75 $\mathrm{ml}$ of $\mathrm{CHCl}_{3}$ and $19.5 \mathrm{ml}$ of $2.0 \mathrm{M} \mathrm{HCOOH}$ (pH 1.56) and the mixture was loaded into the first seven tubes of the distribution apparatus. Number of tubes used in the process was 50. $v_{\mathrm{m}}=2.8 \mathrm{ml}$ (aqueous $\mathrm{HCOOH}), v_{\mathrm{s}}=10.7 \mathrm{ml}\left(\mathrm{CHCl}_{3}\right)$, flow rate $=2 \mathrm{ml} / \mathrm{min}$. (O), experimental curve ob. tained by measuring $A_{400}$ of the effluent fractions. (O), theoretical curve calculated by means of eqns. (4) and (5) (A: $V_{\max }=150$ $\mathrm{ml}, u=49, A_{\max }=22.3$; $\mathrm{B}: V_{\max }=260 \mathrm{ml}$, $n=49, A_{\max }=0.960 ; \mathrm{C}: V_{\max }=295 \mathrm{ml}$, $u=35, \quad A_{\max }=6.46 ; \quad \mathrm{D}: V_{\max }=510 \mathrm{ml}$, $u=37, A_{\max }=1.525 ; \mathrm{E}: V_{\max }=250 \mathrm{ml}$, $\left.u=36, \quad A_{\max }=1.425\right)$. $\mathrm{A}=$ hematopor phyrin, $B=2(4)$-vinyl-4(2)-hydroxyethyldeuteroporphyrin, $\mathrm{C}=$ deuteroporphyrin, $\mathrm{D}=$ mesoporphyrin,$\quad \mathbf{E}=$ protoporphyrin. 
mobile solvent, and the formic acid concentration of this was changed stepwise during the course of the process. This change was effected at the moment when one porphyrin had already emerged with the effluent while the next one to come out was still at the loading end of the apparatus. The theoretical distribution curves were calculated by taking into account the position of the concentration maximum of each porphyrin at the moment the $\mathrm{pH}$ of the mobile solvent was changed. This tube number was substracted from 49 to get the value of $u$ for eqn. (5). $V_{\max }$ for the same equation was obtained by substracting from the total $V_{\max }$ ( $V_{\max }$ in the total volume scale) of the porphyrin in question $V_{\mathrm{m}}$ up to the preceding solvent change (see Fig. 8). The same corrections were made in the calculation of the partition coefficients from eqn. (6). As can be seen from Fig. 8, quite a good separation of five porphyrins can be attained simultaneously with only fifty tubes. However, considerable deviations from theory are observed, especially in the case of proto- and mesoporphyrins. The excessive broadening of the concentration zones, which is probably again caused mainly by association phenomena, impairs the resolution of these porphyrins.

\section{DISCUSSION}

The described experiments show that the aqueous formic acid-chloroform solvent system can be successfully used for the separation of porphyrins by multiple liquid-liquid partition. Some difficulties are encountered, however, with this solvent system. The most serious is undoubtedly the observed excessive broadening of the concentration zones which leads to impaired resolution. Some excess broadening may result from unattained equilibrium between the phases. The main deviations from theory, however, were evidently caused by association in the chloroform phase, which is most convincingly shown in Fig. 5.

The association phenomenon has been overlooked in separations of porphyrins described in the literature. Thus, for example Granick and Bogorad ${ }^{1}$ ascribe the main deviations from theory to porphyrin impurities and do not take association into account. It seems probable that excessive broadening of the concentration zones is caused mainly by the association also in the aqueous hydrochloric acid-diethyl ether solvent system. The fact that the deviations from theory were smaller in the refractionation experiment (Expt. 4 in Ref. 1), where a higher hydrochloric acid concentration was used, parallels the results of the present studies which show that the excessive broadening of the zones could be diminished by lowering the $\mathrm{pH}$ of the upper phase. This effect probably results principally from a change in the porphyrin concentration in the chloroform phase. When the $\mathrm{pH}$ is lowered, more porphyrin becomes protonated and is transferred to the aqueous acid phase where polymerization is not possible. At the same time, the concentration of neutral porphyrin in the chloroform phase is lowered and molecular association is diminished. 
In addition to the lowering of the porphyrin concentration in the organic phase, which is achieved either by lowering the total porphyrin concentration or by lowering the $\mathrm{pH}$ of the aqueous phase, it might also be possible to avoid the association by adding one more component to the solvent system (dimethylformamide, acetone, etc.). However, in that case the selectivity of the solvent system would become impaired, as the mutual solubility of the phases would increase. This was observed by Granick and Bogorad ${ }^{1}$ when they added acetone to the aqueous hydrochloric acid-ether solvent system. A compromise between the dissolving capacity and selectivity of the solvent system may be, however, advantageous, especially if the method is used for preparative purposes.

The selectivity of the aqueous formic acid-chloroform solvent system is considerably better than that of the aqueous hydrochloric acid-ether solvent system with respect to meso-, deutero-, and hematoporphyrins. The separation factor $\left(\beta=\left(k_{1} / k_{2}\right) \geq 1\right)$ has values $6.8-7.4$ for meso- and deuteroporphyrins and 75-80 for deutero- and hematoporphyrins in the aqueous formic acid-chloroform solvent system. The values in the aqueous hydrochloric acid-ether solvent system are 3.5-3.6 and 20-30, respectively. ${ }^{1}$ However, the selectivity with respect to proto- and mesoporphyrins seems to be better in the aqueous hydrochloric acid-ether solvent system. The separation factor for these porphyrins is about 4.5 in the formic acid $(\mathrm{pH} 1.0)$-chloroform system and about 10 in the hydrochloric acid( $\mathrm{pH}$ 1.0)-ether system.4,9

Very useful for the selection of the $\mathrm{pH}$ of the aqueous phase for the fractionation experiments is a plot of $\log K$ against the $\mathrm{pH}$ of the aqueous phase (Fig. 9). The selectivity of the aqueous formic acid-chloroform solvent system can be clearly observed from this plot. The points of Fig. 9 were calculated from the multiple partition data by means of eqn. (6). It can be seen that the theoretically predicted linear relationship between $\log K$ and $\mathrm{pH}$ holds well

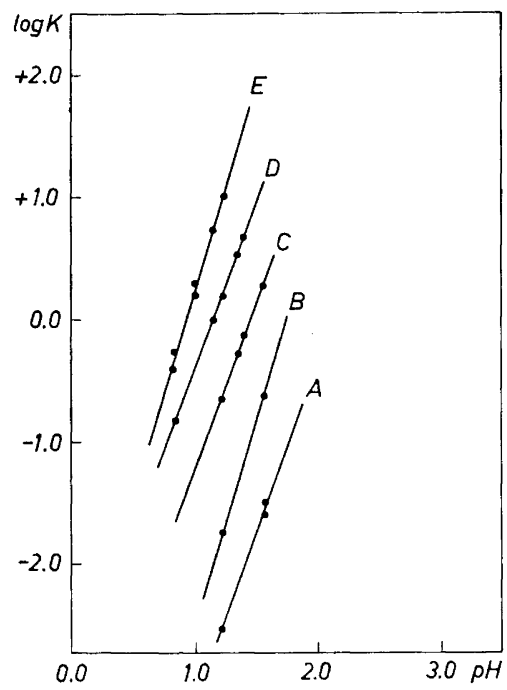

Fig. 9. $\log K$ as a function of $\mathrm{pH}$ of the upper phase. $A=$ hematoporphyrin, $\mathrm{B}=2(4)$ - vinyl - 4(2) - hydroxyethyldeuteroporphyrin, $\mathrm{C}=$ deuteroporphyrin, $\mathrm{D}=$ mesoporphyrin, $\mathbf{E}=$ protoporphyrin.

Acta Chem. Scand. 23 (1969) No. 3 
for the aqueous formic acid-chloroform solvent system. However, the slopes of the lines differ from 2 , most in the case of proto- and 2(4)-vinyl-4(2)-hydroxyethyldeuterophyrins. The slope of the lines of these porphyrins is 3.2 , whereas the slopes of the lines for meso-, deutero-, and hematoporphyrins is about 2.7. These results may be compared with the data given by Herbert ${ }^{9}$ for the aqueous hydrochloric acid-ether solvent system.

The principle of stepwise $\mathrm{pH}$ gradient elution has the following advantages: (1) excessive broadening of the concentration zones is diminished; (2) the solutes are obtained from the apparatus in small volumes of effluent; (3) the fractionation speed can be increased. From these viewpoints the operational partition coefficients ( $k$ values) should be large $(>1)$ in the present fractionation problem. However, in order not to impair the resolution of porphyrins significantly, certain upper limits of $k$ should not be exceeded. These upper limits of $k$ can be calculated for various $\beta$-values from the separation function of the Martin-Synge distribution, ${ }^{12} N=u+1=\left[t^{2}\left(2 \alpha k_{1}+\beta+1\right)^{2} /(\beta-1)^{2}\right]+1$, where $N$ is the minimum number of tubes needed for the separation and $t=\left|t_{1}\right|=\left|t_{2}\right|$ is the abscissa of the standard normal distribution. For the $\beta$ values $4.0,6.0,8.0$, and 10.0 the $k_{1}$ values $3.82,8.90,14.0$, and 19.1 , respectively, are obtained (number of tubes $=50 ; \alpha=0.262$; yield and purity $99.9 \%$ $\left(\left|t_{1}\right|=\left|t_{2}\right|=3.0\right)$; ideal partition). Thus, it can be seen that the resolution of hematoporphyrin and 2(4)-vinyl-4(2)-hydroxyethyldeuteroporphyrin was only partial (Fig. 8), because the operational partition coefficients of these porphyrins were too large $\left(k_{\mathrm{A}}=41.2\right.$ and $\left.k_{\mathrm{B}}=4.27\right)$. The $\mathrm{pH} 1.56$ which was used in this experiment was selected because some solubility difficulties were encountered at higher $\mathrm{pH}$ values with proto- and mesoporphyrins at the beginning of the fractionation. The problem may be solved by adding a small amount of some additional solvent at the beginning of the fractionation, as Granick and Bogorad ${ }^{1}$ have done, or by fractionating hemato- and 2(4)vinyl-4(2)-hydroxyethyldeuteroporphyrins separately afterwards at a higher $\mathrm{pH}$. For meso- and deuteroporphyrins, somewhat lower $\mathrm{pH}$ values than those used seem to be more appropriate. On the basis of Fig. 9, the following $\mathrm{pH}$ values are proposed for the simultaneous separation of five dicarboxylic porphyrins: hematoporphyrin 1.70, 2(4)-vinyl-4(2)-hydroxyethyldeuteroporphyrin 1.50, deuteroporphyrin 1.20 , mesoporphyrin 0.95 , protoporphyrin 0.80 (number of tubes $=50, \alpha=0.262$ ). When stepwise $\mathrm{pH}$ gradient elution is used, it should be noted, in addition, that each porphyrin should be eluted from the apparatus as completely as possible before the $\mathrm{pH}$ of the mobile phase is changed. Otherwise, the tail of a concentration zone may contract and form an additional peak in the effluent curve. Such an additional peak is observed between meso- and deuteroporphyrins in Fig. 8. Under somewhat different conditions, it should be possible to apply the principle of stepwise $\mathrm{pH}$ gradient elution also in CCD.

The two unknown porphyrins which were present in the mesoporphyrin and deuteroporphyrin preparations are probably closely related to each other or identical, because their spectroscopic and partition properties were very similar. The only difference between their absorption spectra is the form of the band at $566-570 \mathrm{~nm}$ which is somewhat wider in the spectrum of the porphyrin from the crude deuteroporphyrin preparation. The fact that the two por-

Acta Chem. Scand. 23 (1969) No. 3 
phyrins were sparingly soluble in the aqueous acid phase (less soluble than protoporphyrin at $\mathrm{pH} 1.22$ ) suggests that these porphyrins are compounds with one or no free carboxyl group.

\section{REFERENCES}

1. Granick, S. and Bogorad, L. J. Biol. Chem. 202 (1953) 781.

2. de la Gandara, J. O. and Torres, J. T. Rec. Trav. Chim. 74 (1955) 637.

3. Paul, K. G. Scand. J. Clin. Lab. Invest. 5 (1953) 212.

4. French, J. M., England, M. T., Lines, J. and Thonger, E. Arch. Biochem. Biophys. 107 (1964) 404.

5. French, J. M. and Thonger, E. Clin. Sci. 31 (1966) 337.

6. Barnes, H. D. S. African J. Lab. Clin. Med. 9 (1963) 177.

7. Willstätter, R. and Mieg, W. Ann. 350 (1906) 1.

8. Zeile, K. and Rau, B. Z. physiol. Chem. 250 (1937) 197.

9. Herbert, F. K. Clin. Chim. Acta 13 (1966) 31.

10. Hietala, P. Ann. Acad. Sci. Fennicae, A II, 100 (1960).

11. Phillips, J. N. Rev. Pure Appl. Chem. (Australia) 10 (1960) 35.

12. Martin, A. J. P. and Synge, R. L. M. Biochem. J. 35 (1941) 1358.

13. Morell, D. B. and Stewart, M. Australian J. Exptl. Biol. Med. Sci. 34 (1956) 211.

14. Muir, H. and Neuberger, A. Biochem. J. 45 (1949) 163.

15. Rampino, L. D. and Nord, F. J. Am. Chem. Soc. 63 (1941) 2745.

16. Schumm, O. Z. physiol. Chem. 178 (1928) 1.

17. Chu, T. C. and Chu, F.-J. J. Am. Chem. Soc. 74 (1952) 6276.

Received July 11, 1968. 